\title{
Clinical physiological parameters for the prediction of gram-negative bacterial infection in the emergency department
}

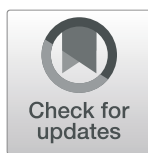

Chan-Peng Hsu ${ }^{1}$, Hsin-Yu Chen², Wei-Lung Chen ${ }^{3,4}$, Jiann-Hwa Chen ${ }^{3,4}$, Chien-Cheng Huang ${ }^{5,6,7}$, Po-Han Wu ${ }^{3 *}$ and Jui-Yuan Chung ${ }^{3,4^{*}}$ (D)

\begin{abstract}
Background: Early detection and treatment of Gram-negative bacteria (GNB), major causative pathogens of sepsis (a potentially fatal condition caused by the body's response to an infection), may benefit a patient's outcome, since the mortality rate increases by 5-10\% for each hour of delayed therapy. Unfortunately, GNB diagnosis is based on bacterial culture, which is time consuming. Therefore, an economic and effective GNB (defined as a positive blood, sputum, or urine culture) infection detection tool in the emergency department (ED) is warranted.

Methods: We conducted a retrospective cohort study in the ED of a university-affiliated medical center between January 01, 2014 and December 31, 2017. The inclusion criteria were as follows: (1) age $\geq 18$; (2) clinical suspicion of bacterial infection; (3) bacterial culture from blood, sputum, or urine ordered and obtained in the ED. Descriptive statistics was performed on patient demographic characteristics, vital signs, laboratory data, infection sites, cultured microorganisms, and clinical outcomes. The accuracy of vital signs to predict GNB infection was identified via univariate logistic regression and receiver operating characteristic (ROC) curve analysis.

Results: A total of 797 patients were included in this study; the mean age was 71.8 years and $51.3 \%$ were male. The odds ratios of patients with body temperature $\geq 38.5^{\circ} \mathrm{C}$, heart rate $\geq 110$ beats per minute, respiratory rate $\geq 20$ breaths per minute, and Glasgow coma scale $(\mathrm{GCS})<14$, in predicting GNB infection were found to be 2.3, 1.4, 1.9, and 1.6, respectively. The area under the curve values for ROC analysis of these measures were $0.70,0.68,0.69$, and 0.67 , respectively.
\end{abstract}

Conclusion: The four physiological parameters were rapid and reliable independent predictors for detection of GNB infection.

Keywords: Clinical parameters, Gram-negative bacteria infection, Emergency department

\footnotetext{
* Correspondence: albertwu0628@gmail.com; bybarian@gmail.com

${ }^{3}$ Department of Emergency Medicine, Chiayi Chang Gung Memorial Hospital, Chiayi 613, Taiwan

Full list of author information is available at the end of the article
}

(C) The Author(s). 2021 Open Access This article is licensed under a Creative Commons Attribution 4.0 International License, which permits use, sharing, adaptation, distribution and reproduction in any medium or format, as long as you give appropriate credit to the original author(s) and the source, provide a link to the Creative Commons licence, and indicate if changes were made. The images or other third party material in this article are included in the article's Creative Commons licence, unless indicated otherwise in a credit line to the material. If material is not included in the article's Creative Commons licence and your intended use is not permitted by statutory regulation or exceeds the permitted use, you will need to obtain permission directly from the copyright holder. To view a copy of this licence, visit http://creativecommons.org/licenses/by/4.0/. The Creative Commons Public Domain Dedication waiver (http://creativecommons.org/publicdomain/zero/1.0/) applies to the data made available in this article, unless otherwise stated in a credit line to the data. 


\section{Background}

Gram-negative bacteria (GNB) are more frequently associated than other microbes with severe sepsis and septic shock [1], a potentially life-threatening consequence of the body's inflammatory response to pathogens. There are two theories regarding how GNB trigger these harmful systemic responses. First, bacteria invade blood vessels and produce inflammatory mediators that circulate throughout the body, resulting in systemic inflammation and multiple organ failure. Second, bacteria and their endotoxins induce local inflammation in extravascular tissues that release immune mediators into the bloodstream [2].

Early recognition of these pathogens and appropriate patient disposition are crucial, as numerous studies have demonstrated benefits to patient outcomes [3-6]. One study indicated that failure to implement effective antibiotic therapy within the first $24 \mathrm{~h}$ in GNB sepsis patients may result in a longer hospital stay and a higher mortality rate [7]. To identify sepsis, Severe Inflammatory Response Syndrome (SIRS) criteria were initially established in 1992 by the American College of Chest Physicians (ACCP), and the Society of Critical Care Medicine (SCCM) defined sepsis with four main variables, including body temperature, heart rate, respiratory rate, white blood cell count, and percentage of band form neutrophils [8]. However, the use of SIRS criteria to identify sepsis has fallen out of favor. The predominant tool in current use is the Sequential Organ Failure Assessment (SOFA), which consists of several complex variables, including mean arterial pressure, consciousness level, partial pressure of oxygen, platelet count, creatinine level, and bilirubin level [8]. The quick sepsisrelated organ failure assessment (qSOFA) score is the prompt and simple version of the SOFA score, which is able to evaluate the patient at bedside. The qSOFA score includes only three clinical parameters: respiratory rate, altered mental status, and systolic blood pressure [8].

The 2018-sepsis-one-hour bundle suggests early administration of one or more broad-spectrum antibiotics, which are able to treat all likely pathogens of patients with suspected infection; antibiotic stewardship was also emphasized as an essential aspect of high-quality sepsis management [9]. At present, the gold standard to diagnose GNB infection is based on bacterial culture, which may take hours or days before the result is known and can lead to delayed treatment decisions. The inflammatory mediators induced by GNB infection may result in a significant inflammatory response, including fever, tachycardia, and shortness of breath [10]. However, none of the scoring tools, such as qSOFA score and SIRS criteria, had ever been used to predict GNB infection. Given that both of the abovementioned scoring tools involve clinical parameters, we aimed to discover a prompt, simple, and inexpensive bedside prediction tool to detect GNB infection in the emergency department (ED) to assist clinical physicians with timely antibiotic treatment and enable accurate patient disposition.

\section{Methods}

\section{Study design, setting, and participants}

This was a retrospective cohort study, conducted in a university-affiliated medical center (Cathay General Hospital, Taipei) in Northern Taiwan with 40 ED beds and 800 ward beds and approximately 55,000 patients visiting annually. The study period was between January 01, 2014 and December 31, 2017. Patients who fulfilled the following inclusion criteria were included: (1) age above 18 years, (2) suspected bacterial infection, and (3) bacterial culture from blood, sputum or urine, ordered and obtained in the ED. Patients who were transferred from other hospitals, had out-of-hospital cardiac arrest, were pregnant, or had mixed infections were excluded.

\section{Definition of variables and primary outcome}

Suspected bacterial infection is identified by: (1) physician's clinical judgment through chart review and infection related disease codes, or (2) ED clinical parameters that indicate infection, such as severe inflammatory response syndrome (SIRS) and qSOFA score. SIRS is defined as a heart rate $>90$ beats per minute, respiratory rate $>20$ breaths per minute, temperature $<36^{\circ} \mathrm{C}$ or $>$ $38^{\circ} \mathrm{C}$, white blood cell count $<4000 / \mathrm{mm}^{3}$ or $>12,000 /$ $\mathrm{mm}^{3}$, and band form $>10 \%$ [11]. qSOFA score is defined as systolic blood pressure $\leq 100 \mathrm{mmHg}$, respiratory rate $\geq$ 22 breaths per minute, and Glasgow Coma Scale < 15 [8]. Positive cultures were defined as: (1) Positive blood culture: at least two bottles of blood culture yielding the same pathogen [12]. Two sets of blood cultures (two aerobic bottles, two anaerobic bottles), were collected from each patient via peripheral venipuncture at two sites, with a 30-min interval between sample collections. (2) Positive sputum culture: pathogen growth in sputum specimens with fewer than 25 squamous epithelial cells per low-power field [13]. Sputum specimen was indicated if a patient had clinical signs, such as productive cough, purulent sputum, dyspnea, or desaturation. Sputum culture was collected by either patient's expectorated sputum in a sterile container after deep coughs, nasotracheal or orotracheal aspiration, or endotracheal tube aspiration. (3) Positive urine culture: pathogen growth $>10^{5}$ colony-forming units (CFU) per milliliter in clean-catch midstream urine specimens [14]. Patients with symptoms of dysuria, frequency, urgency, hematuria, suprapubic pain, fever, chills, or flank pain, with or without costovertebral tenderness, were indicated for urine specimen collection. The methods of urine culture collection included self-collection 
techniques, urethral catheterization, and suprapubic puncture.

Contaminants in cultures were determined as follows: a single positive blood culture with growth of pathogens that represent contamination, including coagulasenegative staphylococci, Corynebacterium species, Bacillus species, Propionibacterium acnes, Micrococcus species, viridans group streptococci, enterococci, and Clostridium perfringens [12]; urine culture with more than two isolates at greater than or equal to $10000 \mathrm{CFU} /$ $\mathrm{mL}$ [14]; positive sputum cultures in which the isolates consisted of normal oral flora, including Neisseria catarrhalis, Candida albicans, diphtheroids, alpha-hemolytic streptococci, and some staphylococci [13].

Sepsis is defined as life-threatening organ dysfunction caused by a dysregulated host response to infection, where organ dysfunction can be identified by sepsisrelated organ failure assessment (SOFA) score $\geq 2$. However, a quick sepsis-related organ failure assessment (qSOFA) score does not require laboratory tests and can be assessed quickly, therefore, in the setting of an emergency department. We used a qSOFA score $\geq 2$ to define sepsis [11].

The diagnosis of pneumonia was based on both clinical presentation of productive cough, chest pain, fever, and dyspnea, and pulmonary infiltration visible on the chest image. The indications for chest radiographies were based on both physician's clinical judgement and patient's clinical presentation, including productive cough, chest pain, fever, and dyspnea.

\section{Data collection and assignment to case and control groups}

The retrospective chart review method was used to acquire data of patients who fulfilled the inclusion criteria. Demographic characteristics, including vital signs (obtained at the ED triage), laboratory data, infection sites, cultured microorganisms, qSOFA scores, SIRS criteria, and clinical outcomes, were obtained by an emergency physician (Table 1). In total, 903 bacteria-infected ED patients were initially recruited, with a total of 797 patients included (174 culture negative patients and 623 culture positive patients) in the study. Exclusions (106 patients) were made for insufficient data, presence of mixed infections, occurrence of an out-of-hospital cardiac arrest, transferal of patients treated at other hospitals, or pregnant patients (Fig. 1). The recruited patients were further divided into two groups based on the culture result, with 278 patients assigned to the GNB group and 519 patients to the non-GNB group (including 174 culture negative patients). All variables were compared between the two groups, and the accuracy of clinical parameters to predict GNB infection were also analyzed.

\section{Ethical statement}

This study was approved by the institutional Review Board of the Cathay General Hospital and was conducted according to the Declaration of Helsinki. This was an observational study, and the patients' data were fully anonymized; the need for informed consent from the patients was waived.

\section{Statistical analysis}

We used SPSS 23.0 for Mac (SPSS Inc., Chicago, IL, USA) to perform statistical analysis. Continuous normally distributed data are presented as mean \pm standard deviation (SD), while continuous data that were not normally distributed are presented as median $+/$ - interquartile range (IQR). Categorical variables were presented as percentages. Independent samples $t$-test, the MannWhitney, or Wilcoxon test were used to analyze continuous variables. Pearson's chi-square test or Fisher's exact test was used for categorical variables. Univariate analysis was performed to evaluate the prediction of GNB infection among the four clinical parameters that showed significant difference $(p<0.05)$ between the GNB and the non-GNB group (Table 2). We calculated the odds ratio of GNB infection in patients with suspected bacterial infection, using 2, 3, or 4 positive clinical parameters. The optimal cut-off point of each clinical parameter used to predict GNB infection was calculated via Youden index. The area under the receiver operating characteristic curve (AUROC) was then used to evaluate GNB prediction discrimination ability (Table 3). Sensitivity, specificity, positive predictive value, and negative predictive value were also calculated to determine the diagnostic accuracy of the four clinical parameters in predicting GNB infection (Table 4).

\section{Results}

A total of 797 patients were included in this study. The male to female ratio was approximately equal. The mean \pm SD age was $71.8 \pm 17.2$ years, and the GNB group was older than the non-GNB group at $77.0 \pm 1.2$ and $69.0 \pm 17.5$ years, respectively. Glasgow coma scale (GCS), heart rate, respiratory rate, and body temperature were significantly different ( $p$-value $<0.01,0.02,<0.01$, 0.01 ) between GNB and non-GNB group. The mean \pm SD of GCS was lower in the GNB group $(10.9 \pm 3.8)$ than the non-GNB group $(11.9 \pm 4.0)$. Heart rate, respiratory rate, and body temperature were higher in the GNB group than the non-GNB group for $105.0 \pm 25.2,21.9 \pm$ $6.6,37.7 \pm 1.5$, and $100.0 \pm 29.5,20.3 \pm 6.9,37.0 \pm 4.3$, respectively.

Meanwhile, significant elevation of heart rate, respiratory rate and body temperature were noted in the culture positive patients $(102.3 \pm 27.2,22.0 \pm 5.8,37.3 \pm 4.5)$ than the culture negative patients $(97.8 \pm 34.0,20.1 \pm 9.9$, 
Table 1 Demographics of emergency department adult patients with suspected bacterial infection

\begin{tabular}{|c|c|c|c|c|}
\hline Characteristics & $\begin{array}{l}\text { Total patients } \\
(n=797)\end{array}$ & $\begin{array}{l}\text { GNB } \\
(n=278)\end{array}$ & Non-GNB $^{\mathrm{a}}(n=519)$ & $p$-value \\
\hline Age (mean \pm SD) & $71.8 \pm 17.2$ & $77.0 \pm 1.2$ & $69.0 \pm 17.5$ & $<0.01$ \\
\hline Male (\%) & $51.3 \%$ & $47.5 \%$ & $53.4 \%$ & 0.11 \\
\hline \multicolumn{5}{|l|}{ Vital signs (mean \pm SD) } \\
\hline Glasgow coma scale & $11.5 \pm 4.0$ & $10.9 \pm 3.8$ & $11.9 \pm 4.0$ & $<0.01$ \\
\hline $\mathrm{SBP}(\mathrm{mmHg})$ & $96.0 \pm 37.3$ & $98.0 \pm 35.0$ & $94.9 \pm 38.5$ & 0.26 \\
\hline Heart rate (n/min) & $101.7 \pm 28.1$ & $105.0 \pm 25.2$ & $100.0 \pm 29.5$ & 0.02 \\
\hline Respiratory rate (n/min) & $20.8 \pm 6.8$ & $21.9 \pm 6.6$ & $20.3 \pm 6.9$ & $<0.01$ \\
\hline Body temperature $\left({ }^{\circ} \mathrm{C}\right)$ & $37.3 \pm 3.6$ & $37.7 \pm 1.5$ & $37.0 \pm 4.3$ & 0.01 \\
\hline \multicolumn{5}{|l|}{ Laboratory data (median, IQR) } \\
\hline WBC $\left(10^{3} / \mathrm{mm}^{3}\right)$ & $11.6(8.1-16.6)$ & $12.3(8.8-17.1)$ & $11.4(7.9-16.4)$ & 0.13 \\
\hline CRP (mg/dL) & $6.6(2.0-14.8)$ & $7.9(3.1-17.4)$ & $5.5(1.3-12.9)$ & $<0.01$ \\
\hline \multicolumn{5}{|l|}{ Infection sites (\%) } \\
\hline Pneumonia & $26.3 \%$ & $37.1 \%$ & $20.6 \%$ & $<0.01$ \\
\hline Urinary tract infection & $22.1 \%$ & $55.8 \%$ & $4.0 \%$ & $<0.01$ \\
\hline Intra-abdominal infection & $11.4 \%$ & $17.3 \%$ & $8.3 \%$ & $<0.01$ \\
\hline Soft tissue infection & $3.1 \%$ & $3.2 \%$ & $3.1 \%$ & 0.90 \\
\hline Infectious endocarditis & $0.8 \%$ & $1.4 \%$ & $0.4 \%$ & 0.10 \\
\hline CNS infection & $0.1 \%$ & $0.4 \%$ & $0 \%$ & 0.17 \\
\hline HIV infection & $0.4 \%$ & $0.0 \%$ & $0.6 \%$ & 0.20 \\
\hline Bacteremia & $14.3 \%$ & $36.7 \%$ & $23.3 \%$ & $<0.01$ \\
\hline \multicolumn{5}{|l|}{ Medical history (\%) } \\
\hline Diabetes & $13.2 \%$ & $11.9 \%$ & $13.9 \%$ & 0.43 \\
\hline Malignancy & $18.1 \%$ & $16.5 \%$ & $18.9 \%$ & 0.41 \\
\hline Chronic kidney disease & $12.5 \%$ & $6.1 \%$ & $12.0 \%$ & $<0.01$ \\
\hline Uremia under hemodialysis & $2.9 \%$ & $0.7 \%$ & $4.9 \%$ & $<0.01$ \\
\hline COPD & $1.6 \%$ & $2.2 \%$ & $1.3 \%$ & 0.39 \\
\hline Liver cirrhosis & $4.9 \%$ & $3.6 \%$ & $5.6 \%$ & 0.21 \\
\hline Autoimmune disease & $2.0 \%$ & $2.2 \%$ & $1.9 \%$ & 0.82 \\
\hline qSOFA $\geq 2(\%)$ & $42.7 \%$ & $56.6 \%$ & $46.4 \%$ & $<0.01$ \\
\hline SIRS $\geq 3(\%)$ & $29.7 \%$ & $39.6 \%$ & $24.5 \%$ & $<0.01$ \\
\hline Mortality (\%) & $26.7 \%$ & $27.7 \%$ & $24.8 \%$ & 0.37 \\
\hline
\end{tabular}

GNB gram-negative bacteria, $S D$ standard deviation, $S B P$ systolic blood pressure, IQR interquartile range, WBC white blood cell, CRP C-reactive protein, CNS central nervous system, HIV Human immunodeficiency virus, COPD chronic obstructive pulmonary disease, qSOFA quick sepsis related organ failure assessment, SIRS systemic inflammatory response syndrome

ancluding culture negative patients

$36.4 \pm 3.1$; GCS was $11.2 \pm 4.3$ in the culture positive patients, which was significantly lower than the culture negative patients. Lower systolic blood pressure was also noted in the culture positive patients than the culture negative patients for $91.4 \pm 42.1$ and $96.0 \pm 37.2$ (Supplementary Table 1).

The median C-reactive protein (CRP) was 7.9 (IQR: 3.1-17.4) in the GNB group, which was higher than that in the non-GNB group, 5.5 (IQR: 1.3-12.9). The prevalence of pneumonia, urinary tract infection, intra-abdominal infection, and bacteremia was moderately higher in the GNB group than in the non-GNB group at 37.1, 55.8, 17.3 , and $36.7 \%$, respectively. The percentage of qSOFA score $\geq 2$ and SIRS criteria $\geq 3$ were both significantly higher in the GNB group, at 50.7 and $39.6 \%$, respectively, than the non-GNB group, at 38.3 and $24.5 \%$, respectively. The most frequently identified bacterium from the blood and the urine specimen was Escherichia coli (38\%); while the most frequently identified pathogen from the sputum was Pseudomonas aeruginosa (40\%) (Fig. 2). 


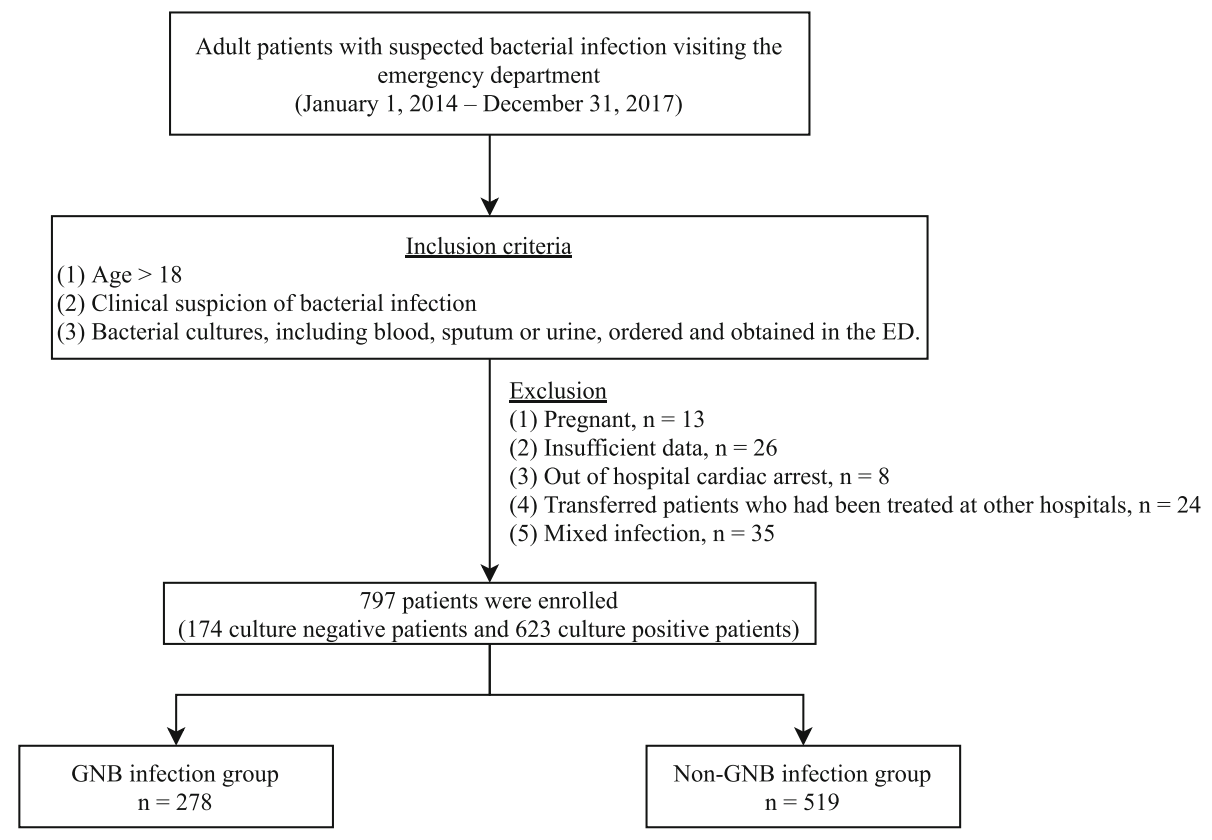

Fig. 1 Flowchart of this study

Comparing bacteremic patients with non-bacteremic patients, regardless of GNB status, bacteremic patients had a significantly higher heart rate, respiratory rate, and body temperature than non-bacteremic patients at $105.3 \pm 24.2,21.6 \pm 6.5$, and $37.8 \pm 1.4$, respectively. The mortality percentage in bacteremic patients, regardless of GNB status, was $24.0 \%$; it was $28.2 \%$ for the nonbacteremic patients, $p$-value $=0.22$. Bacteremic patients had significantly higher percentages for quick sepsisrelated organ failure assessment (qSOFA) score $\geq 2$ and severe inflammatory response syndrome (SIRS) $\geq 3$ at 47.3 and $38.5 \%$, respectively, than the non-bacteremic patients at 40.1 and $25.1 \%$, respectively (Supplementary Table 2). Results showed a higher rate of pneumonia, urinary tract infection, soft tissue infection, and

Table 2 Predicting gram-negative bacterial infection by specific clinical physiology parameters, quick sepsis related organ failure assessment score and systemic inflammatory response syndrome criteria of adult patients with suspected bacterial infection in emergency department, identified by logistic regression

\begin{tabular}{llll}
\hline & Odds ratio & $\mathbf{9 5 \% ~ C l}$ & $\boldsymbol{p}$-value \\
\hline $\mathrm{BT} \geq 38.5^{\circ} \mathrm{C}$ & 2.30 & $1.90-3.83$ & $<0.01$ \\
$\mathrm{RR} \geq 20 / \mathrm{min}$ & 1.91 & $1.51-2.92$ & $<0.01$ \\
$\mathrm{GCS}<14$ & 1.57 & $1.38-2.50$ & $<0.01$ \\
$\mathrm{HR} \geq 110$ beats/min & 1.38 & $1.27-2.30$ & 0.04 \\
$\mathrm{qSOFA} \geq 2$ & 1.47 & $1.07-1.97$ & 0.01 \\
$\mathrm{SIRS} \geq 3$ & 1.81 & $1.33-2.55$ & $<0.01$
\end{tabular}

$B T$ body temperature, $R R$ respiratory rate, GCS Glasgow coma scale, $H R$ heart rate bacteremia in septic patients than in non-septic patients. Furthermore, mortality was significantly higher in the septic patients at $55.4 \%$, than in the non-septic patients at $38.0 \%$ (Supplementary Table 3).

The mortality percentage was higher in the GNB group $(27.7 \%)$ than the non-GNB group (24.8\%). The optimal cut-off point for the clinical parameters to predict GNB infection among ED adult bacteria-infected patients, calculated via Youden index, are as follows: body temperature $\geq 38.5^{\circ} \mathrm{C}$, heart rate $\geq 110$ beats per minute, respiratory rate $\geq 20$ breaths per minute, and $\mathrm{GCS}<14$. Logistic regression showed that patients with each of these parameters had, respectively, a 2.3-, 1.4-, 1.9-, and 1.6-fold greater risk of GNB infection than the patients who did not meet the cut-off point of the four study clinical parameters (multivariate analyses showed that patients with body temperature $\geq 38.5^{\circ} \mathrm{C}$, heart rate $\geq 110$ beats per minute, respiratory rate $\geq 20$ breaths per minute, and GCS $<14$ had a 2.44-,1.36-, 1.92-, and 0.73 -fold greater risk of GNB infection than the patients

Table 3 Adjusted AUROC for gram-negative bacterial infection prediction by clinical physiology parameters of adult patients with suspected bacterial infection in emergency department

\begin{tabular}{llll}
\hline & AUROC & $\mathbf{9 5 \% ~ C l}$ & $\boldsymbol{p}$-value \\
\hline $\mathrm{BT} \geq 38.5^{\circ} \mathrm{C}$ & 0.70 & $0.66-0.74$ & $<0.01$ \\
$\mathrm{RR} \geq 20 / \mathrm{min}$ & 0.69 & $0.65-0.72$ & $<0.01$ \\
$\mathrm{GCS}<14$ & 0.68 & $0.64-0.72$ & $<0.01$ \\
$\mathrm{HR} \geq 110$ beats/min & 0.67 & $0.63-0.71$ & $<0.01$
\end{tabular}

AUROC Area under the Receiver Operating Characteristic, $B T$ body temperature, $R R$ respiratory rate, GCS Glasgow coma scale, $H R$ heart rate 
Table 4 Performance of the clinical physiology parameters in predicting gram-negative bacterial infection in adult patients with suspected bacterial infection in emergency department

\begin{tabular}{lllll}
\hline & Sensitivity & Specificity & Positive predictive value & Negative predictive value \\
\hline $\mathrm{BT} \geq 38.5^{\circ} \mathrm{C}$ & $0.32(0.26-0.37)$ & $0.85(0.82-0.88)$ & $0.54(0.46-0.61)$ & $0.70(0.66-0.74)$ \\
$\mathrm{RR} \geq 20$ breaths/min & $0.50(0.44-0.56)$ & $0.67(0.63-0.71)$ & $0.45(0.39-0.50)$ & $0.71(0.67-0.75)$ \\
$\mathrm{GCS}<14$ & $0.60(0.54-0.66)$ & $0.55(0.51-0.59)$ & $0.42(0.37-0.47)$ & $0.72(0.68-0.77)$ \\
$\mathrm{HR} \geq 110$ beats/min & $0.45(0.39-0.51)$ & $0.67(0.64-0.72)$ & $0.43(0.37-0.48)$ & $0.70(0.66-0.74)$ \\
\hline
\end{tabular}

$B T$ body temperature, $R R$ respiratory rate, GCS Glasgow coma scale, $H R$ heart rate

who did not meet the cut-off point of the four study clinical parameters [Supplementary Table 4]). Meanwhile, qSOFA $\geq 2$ and SIRS $\geq 3$ had a 1.47 - and 1.81-fold greater risk of GNB infection, respectively (Table 2). Diagnostic accuracy tests of the four clinical parameters in predicting GNB infection showed GCS $<14$ with the highest sensitivity and negative predictive values of 0.6 and 0.72 , respectively; while $\mathrm{BT} \geq 38.5^{\circ} \mathrm{C}$ had the highest specificity and positive predictive values of 0.85 and 0.54 , respectively. All four clinical parameters had approximately equal negative predictive values of 0.70 0.72 (Table 4$)$. The AUROC, adjusted by age $(p<0.01)$, chronic kidney disease (CKD) $(p<0.01)$ and uremia under dialysis, in predicting GNB infection among ED adult bacteria-infected patients, showed body temperature $\geq 38.5^{\circ} \mathrm{C}$ and had an acceptable discrimination ability at $0.7(0.66-0.74)$ (Fig. 3). We calculated the odds ratio of GNB infection in suspected bacterial infection patients with 2,3 , or 4 of the study clinical parameters showing positive results. Results showed an
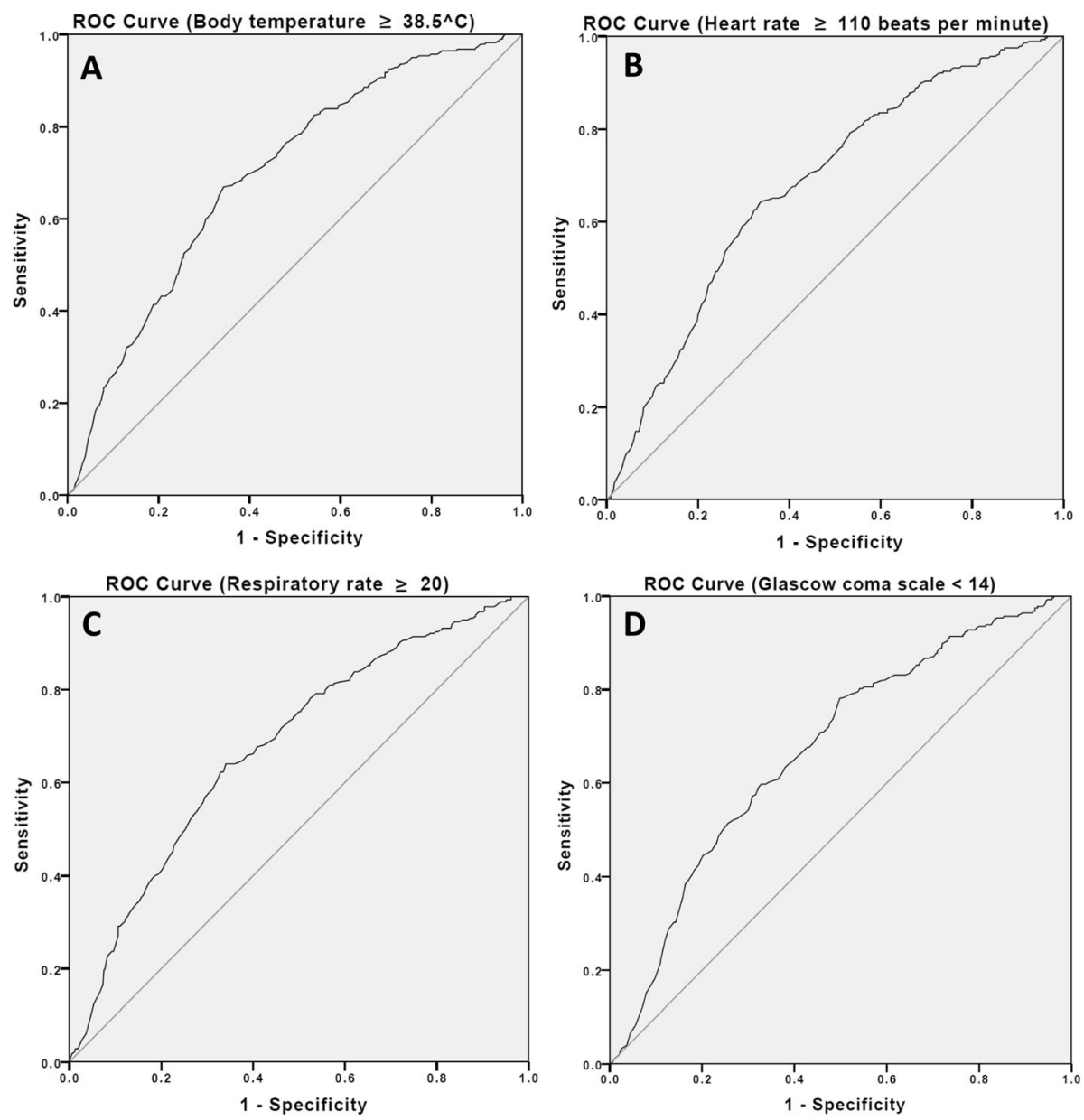

Fig. 2 a. Area under the curve of body temperature $\geq 38.5^{\circ} \mathrm{C} ; \mathbf{b}$. heart rate $\geq 110$ beats per minute; $\mathbf{c}$. respiratory rate $\geq 20$ breaths per minute; $\mathbf{d}$. Glasgow coma scale $<14$ to predict gram-negative bacterial infection in the emergency department 


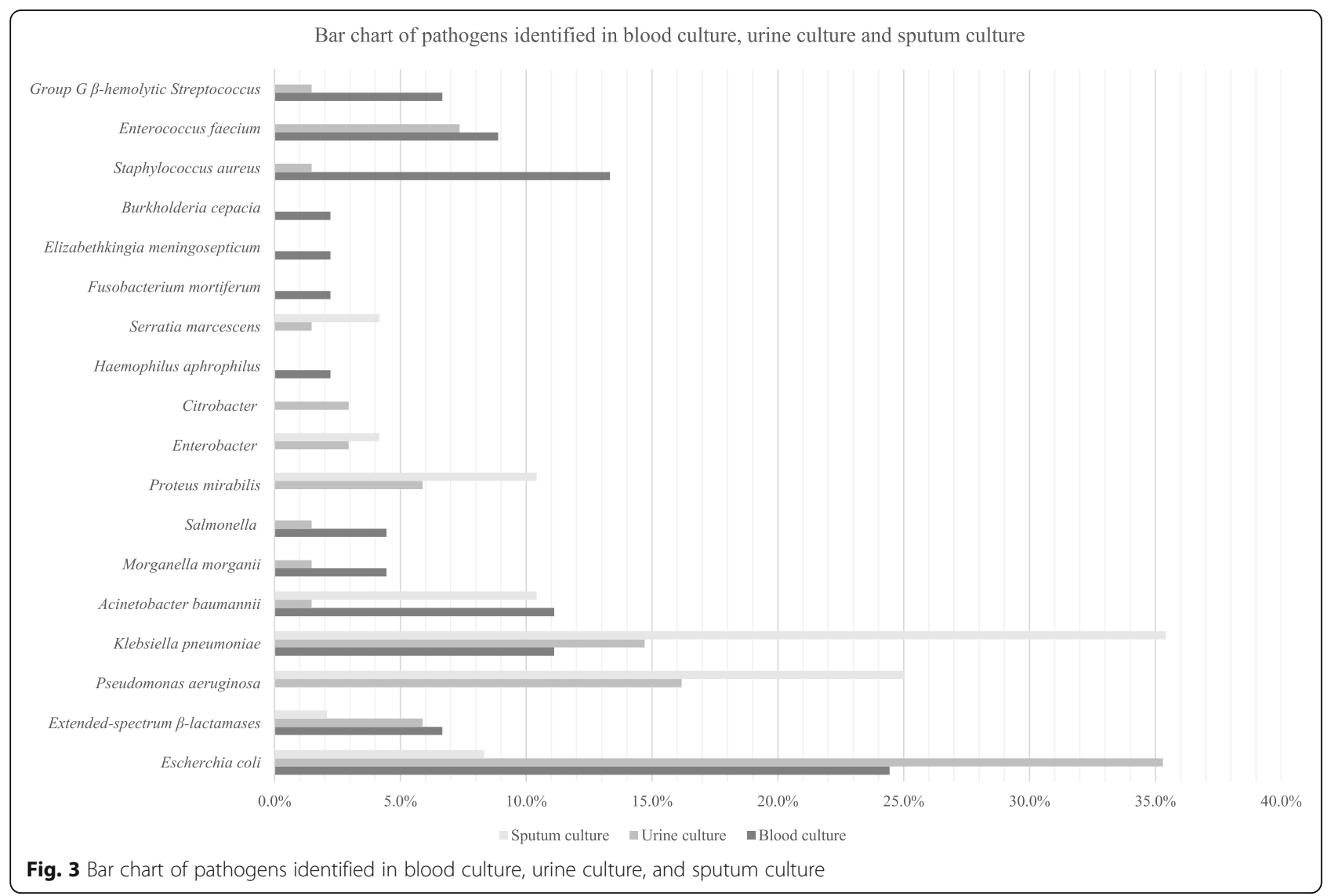

increasing trend of the odds ratio as the numbers of the study clinical parameters that were positive increased. The odds ratios of GNB infection were 1.79, 2.29, and 4.32 in patients with 2,3 , or 4 of the study clinical parameters showing positive, respectively (Supplementary Table 5). While the odds ratio of GNB combined with sepsis in patients with 2,3 , or 4 of the study indicators showing positive results were 4.02, 4.83, and 5.50, respectively (Supplementary Table 6).

\section{Discussion}

It is crucial to quickly identify the pathogens in septic patients and make a prompt decision in a busy ED, as the mortality rate may increase up to $10 \%$ with every hour of delayed diagnosis [15]. Unlike the timeconsuming blood culture, assessment of clinical physiological parameters has the advantage of being immediate and easy to acquire in the ED. SIRS criteria and qSOFA scores were both used to identify sepsis via clinical parameters. However, the relative accuracy of these tools for the identification of sepsis remains under debate [16], and none of these have been used to predict GNB infection yet.

This study identified the key clinical physiological parameters that could predict GNB infection in the ED by reviewing medical records of adult patients with suspected bacterial infection, according to the definition mentioned in the Methods. Although two of the qSOFA score variables, GCS and respiratory rate, and three of the SIRS criteria variables, heart rate, body temperature, and respiratory rate, were correlated to GNB infection, the cut-off points were different from the qSOFA score and SIRS criteria, as the clinical parameters that predicted GNB infection were: body temperature $38.5^{\circ} \mathrm{C}$, heart rate $\geq 110$ beats per minute, respiratory rate $\geq 20$ breaths per minute, and GCS $<14$. These further indicate that GNB infection may stimulate both severe inflammatory response (body temperature $\geq 38.5^{\circ} \mathrm{C}$, heart rate $\geq 110$ beats per minute, respiratory rate $\geq 20$ breaths per minute) and cause organ dysfunction $(\mathrm{GCS}<14)$. We determined that the cut-off points of body temperature of $\geq 38.5^{\circ} \mathrm{C}$ and heart rate $\geq 110$ beats per minute were higher than the SIRS criteria of $>38^{\circ} \mathrm{C}$ and $>90$ beats per minute. These were probably related to the systemic inflammation, which could include effects induced by the GNB, such as increased vascular permeability, leukocyte-endothelial adhesion, and neuroendocrine dysregulation [2]. The circulating GNB endotoxin may further precipitate organ dysfunction and shock status, with consequences of poor perfusion to the 
central nervous system and changes in GCS [2]. In this study and other reports, the respiratory tract was one of the most common infection sites for GNB [17], resulting in pneumonia and increased respiratory rates. Despite the fact that GNB infection may be more likely to develop into sepsis, $43.3 \%$ of the GNB infected patients did not fulfill the criteria of $q S O F A \geq 2$. Furthermore, the odds ratio of qSOFA $\geq 2$ in predicting GNB infection was 1.47, which was lower than the individual clinical parameters of body temperature $\geq 38.5^{\circ} \mathrm{C}$, respiratory rate $\geq 20$ breaths per minute, and GCS $<14$, at $2.30,1.91$, and 1.57, respectively. Although both GNB and non-GNB groups included substantial numbers of patients with elevated sepsis scores and fatal outcomes, the GNB group has statistically significantly greater disease severity than the non-GNB group, as higher percentage of qSOFA $\geq 2$ and SIRS $\geq 3$ were noted in the GNB group.

Prior medical history did not show a significant difference between the GNB and the non-GNB group, making it difficult to predict GNB infection using these methods. However, higher prevalence of CKD and uremia under dialysis were noted in the non-GNB group. A similar finding was noted in a previous study conducted by Berman and colleagues, who concluded that instead of GNB, the major pathogens in infected dialysis patients were gram-positive cocci [18]. In patients with CKD, Escherichia coli and Staphylococcus aureus accounted for the majority of bloodstream infections, while Staphylococcus aureus was especially prominent in CKD patents with an estimated glomerular filtration rate $<30$ $\mathrm{mL}$ per minute per $1.73 \mathrm{~m}^{2}$ [19].

This study demonstrated a significantly higher CRP level in the GNB group than the non-GNB group. Ryuzo and colleagues discovered a similar result, where CRP and interleukin-6 were higher in gramnegative bacteremia than in gram-positive bacteremia in intensive care unit patients [1]. Another study also showed CRP and procalcitonin as effective predictors of bloodstream GNB infection [20]. Although laboratory data may act as potential indicators of GNB infection, it still requires time to obtain the result, and this may jeopardize the early treatment strategy. Furthermore, despite several newly developed molecular rapid diagnostic tools being available for the early detection of these pathogen species, these tools are not widely used in most medical facilities due to their unavailability and expense [21-24].

We discovered that GCS $<14$ had the highest sensitivity at 0.6 , and $\mathrm{BT} \geq 38.5^{\circ} \mathrm{C}$ had the highest specificity at 0.85 . The negative predictive values of the four study clinical parameters were $0.70-0.72$, which indicated that patients who did not meet the cut-off point of the four study clinical parameters were less likely to be infected with GNB. Furthermore, the risk of GNB infection may increase by 4 -fold, while when 2, 3, or 4 of the study clinical parameters showed positive, the odds ratios were $1.79,2.29$, and 4.32 , respectively. The need to treat for sepsis as well as GNB infection should be considered in patients with 2,3 , or 4 of the study clinical parameters showing positive results, as the odds ratios were 4.02, 4.83 , and 5.50 , respectively.

To our knowledge, this was the first study conducted to evaluate physiological parameters to predict GNB infection in the ED. However, this study also has some limitations. First, some valuable information and data may be missing due to the nature of a retrospective study setting. Furthermore, due to the retrospective setting of this study, much greater heterogeneity of clinical and laboratory findings was noted in the non-GNB group than the GNB group. It is also difficult to ensure external validity for the larger population of GNB infection, that further studies are needed to validate the results of this study. Second, this was a single-center study and thus the bacterial species detected may have a selection bias. Third, the study was conducted in a tertiary medical center, where the disease severity of the visiting patients may be higher. Fourth, several unmeasured confounding factors may exist, such as the severity of underlying diseases and the time of treatment. Fifth, clinical parameters were obtained only once after arrival at the ED, while initial vital signs may better reflect the original patient status rather than the vital signs obtained after treatment. Sixth, false negative cases may be left out; however, the false negative rate was very low, as Peretz and colleagues discovered that only $0.13 \%$ of the initially negative blood cultures were positive after further examination by Gram staining [25]. Although the odds ratio for any combination of the two or three clinical parameters to predict GNB infection were provided in the Supplementary Table 5, we did not calculate the odds ratio for each possible combinations of the two-parameter or three parameter model (there are six possible twoparameter combinations and four possible threeparameter combinations), therefore, developing a clinical physiology scoring tool to predict GNB infection based on the findings of this research and comparing the newly established tool with the existing scoring systems, such as qSOFA and SIRS criteria, will be our future goal.

\section{Conclusion}

Clinical physiological parameters, including body temperature $\geq 38.5^{\circ} \mathrm{C}$, heart rate $\geq 110$ beats per minute, respiratory rate $\geq 20$ breaths per minute, and GCS $<14$, were four rapid, simple, and cost-effective independent predictors that could detect GNB infection early in the ED. 


\section{Supplementary Information}

The online version contains supplementary material available at https://doi. org/10.1186/s12879-020-05758-1.

Additional file 1: Table S1. Demographics of culture positive and culture negative patients among those with suspected bacterial infection in the emergency department. Table S2. Demographics of bacteremic and non-bacteremic patients among those with suspected bacterial infection in the emergency department. Table S3. Demographics of septic and non-septic patients among those with suspected bacterial infection in the emergency department. Table S4. Multivariate analysis of specific clinical physiology parameters predicting gram-negative bacterial infection in adult patients with suspected bacterial infection in the emergency department. Table S5. Logistic regression analysis identifying the number of positive clinical physiology parameters predicting gram-negative bacterial infection in adult patients with suspected bacterial infection in the emergency department. Table S6. Logistic regression analysis identifying the number of positive clinical physiology parameters predicting gram-negative bacterial infection with sepsis in adult patients with suspected bacterial infection in the emergency department.

\section{Abbreviations}

GNB: Gram-negative bacteria; SIRS: Severe Inflammatory Response Syndrome; ACCP: American College of Chest Physicians; SCCM: Society of Critical Care Medicine; SOFA: Sequential Organ Failure Assessment; qSOFA: Quick sepsisrelated organ failure assessment; ED: Emergency department; CFU: Colonyforming unit; SD: Standard deviation; IQR: Interquartile range; AUROC: Area under the receiver operating characteristic curve; GCS: Glasgow coma scale; CRP: C-reactive protein; CKD: Chronic kidney disease

\section{Acknowledgments}

We thank Editage for English language editing.

\section{Code availability}

Not applicable.

\section{Authors' contributions}

$\mathrm{CPH}, \mathrm{HYC}$, WLC, JHC, CCH, PHW, and JYC designed and conceived this study, HYC and CPH wrote the manuscript. JYC performed the statistical analysis. JHC, WLC, and CCH provided professional suggestions. All authors read and approved the final manuscript.

\section{Funding}

Not applicable.

\section{Availability of data and materials}

All data generated or analyzed during this study were included in this manuscript.

\section{Ethics approval and consent to participate}

This study was approved by the institutional Review Board of the Cathay General Hospital and was conducted according to the Declaration of Helsinki. Due to the retrospective nature of this study, the need for informed consent from the patients was not necessary.

\section{Consent for publication}

Not applicable.

\section{Competing interests}

All authors denied any conflict of interest.

\section{Author details}

'Department of Emergency Medicine, Hsinchu Cathay General Hospital, Hsinchu, Taiwan. ²Department of Pediatric, Hsinchu Cathay General Hospital, Hsinchu, Taiwan. ${ }^{3}$ Department of Emergency Medicine, Chiayi Chang Gung Memorial Hospital, Chiayi 613, Taiwan. ${ }^{4}$ Fu Jen Catholic University School of Medicine, Taipei, Taiwan. ${ }^{5}$ Department of Emergency Medicine, Chi-Mei Medical Center, Tainan, Taiwan. ${ }^{6}$ Department of Environmental and Occupational Health, College of Medicine, National Cheng Kung University,
Tainan, Taiwan. ${ }^{7}$ Department of Senior Services, Southern Taiwan University of Science and Technology, Tainan, Taiwan.

Received: 7 May 2020 Accepted: 30 December 2020

Published online: 13 January 2021

\section{References}

1. Abe R, Oda S, Sadahiro T, et al. Gram-negative bacteremia induces greater magnitude of inflammatory response than gram-positive bacteremia. Crit Care. 2010;14(2):R27.

2. Munford RS. Severe sepsis and septic shock: the role of gram-negative bacteremia. Annu Rev Pathol. 2006;1:467-96.

3. Micek ST, Welch EC, Khan J, et al. Empiric combination antibiotic therapy is associated with improved outcome against sepsis due to gram-negative bacteria: a retrospective analysis. Antimicrob Agents Chemother. 2010;54(5): $1742-8$

4. Perez KK, Olsen RJ, Musick WL, et al. Integrating rapid pathogen identification and antimicrobial stewardship significantly decreases hospital costs. Arch Pathol Lab Med. 2013;137(9):1247-54.

5. Zasowski EJ, Claeys KC, Lagnf AM, Davis SL, Rybak MJ. Time is of the essence: the impact of delayed antibiotic therapy on patient outcomes in hospital-onset enterococcal bloodstream infections. Clin Infect Dis. 2016; 62(10):1242-50

6. Marchaim D, Gottesman T, Schwartz O, et al. National multicenter study of predictors and outcomes of bacteremia upon hospital admission caused by Enterobacteriaceae producing extended-spectrum beta-lactamases. Antimicrob Agents Chemother. 2010;54(12):5099-104.

7. Shorr AF, Micek ST, Welch EC, et al. Inappropriate antibiotic therapy in gram-negative sepsis increases hospital length of stay. Crit Care Med. 2011; 39(1):46-51.

8. Singer M, Deutschman CS, Seymour CW, et al. The third international consensus definitions for Sepsis and septic shock (Sepsis-3). JAMA. 2016; 315(8):801-10. https://doi.org/10.1001/jama.2016.0287.

9. Levy MM, Evans LE, Rhodes A. The surviving sepsis campaign bundle: 2018 update. Intensive Care Med. 2018:44:925-8.

10. Chou HL, Han ST, Yeh CF, et al. Systemic inflammatory response syndrome is more associated with bacteremia in elderly patients with suspected sepsis in emergency departments. Medicine (Baltimore). 2016;95(49):e5634.

11. Bone RC, Balk RA, Cerra FB, et al. Definitions for sepsis and organ failure and guidelines for the use of innovative therapies in sepsis. The ACCP/SCCM Consensus Conference Committee. American College of Chest Physicians/ Society of Critical Care Medicine. Chest. 1992;101(6):1644-55.

12. Weinstein MP. Current blood culture methods and systems: clinical concepts, technology, and interpretation of results. Clin Infect Dis. 1996:23(1):40-6.

13. Joyce SM. Sputum analysis and culture. Ann Emerg Med. 1986;15(3):325-8.

14. Bekeris LG, Jones BA, Walsh MK, Wagar EA. Urine culture contamination: a College of American Pathologists Q-Probes study of 127 laboratories. Arch Pathol Lab Med. 2008;132(6):913-7.

15. Lin J, Chen L, Lin J, et al. A novel approach for fast detection of sepsis with gram-negative bacterial infection. Microb Biotechnol. 2018;11(6):1121-3.

16. Marik PE, Taeb AM. SIRS, qSOFA and new sepsis definition. J Thorac Dis. 2017;9(4):943-5.

17. Rodrigo-Troyano A, Sibila O. The respiratory threat posed by multidrug resistant gram-negative bacteria. Respirology. 2017;22(7):1288-99.

18. Berman SJ, Johnson EW, Nakatsu C, et al. Burden of infection in patients with end-stage renal disease requiring long-term dialysis. Clin Infect Dis. 2004:39(12):1747-53

19. James MT, Laupland KB, Tonelli M, et al. Risk of bloodstream infection in patients with chronic kidney disease not treated with dialysis. Arch Intern Med. 2008;168(21):2333-9.

20. Gutiérrez-Gutiérrez B, Morales I, Pérez-Galera S, et al. Predictive value of the kinetics of procalcitonin and C-reactive protein for early clinical stability in patients with bloodstream infections due to gram-negative bacteria. Diagn Microbiol Infect Dis. 2019:93(1):63-8.

21. Clerc O, Prod'hom G, Vogne C, et al. Impact of matrix-assisted laser desorption ionization time-of-flight mass spectrometry on the clinical management of patients with gram-negative bacteremia: a prospective observational study. Clin Infect Dis. 2013:56(8):1101-7.

22. Banerjee $R$, Teng CB, Cunningham SA, et al. Randomized trial of rapid multiplex polymerase chain reaction-based blood culture identification and susceptibility testing. Clin Infect Dis. 2015;61(7):1071-80. 
23. Dodémont M, De Mendonça R, Nonhoff C, Roisin S, Denis O. Performance of the Verigene gram-negative blood culture assay for rapid detection of bacteria and resistance determinants. J Clin Microbiol. 2014;52(8):3085-7.

24. Hill JT, Tran KD, Barton KL, Labreche MJ, Sharp SE. Evaluation of the nanosphere Verigene BC-GN assay for direct identification of gram-negative bacilli and antibiotic resistance markers from positive blood cultures and potential impact for more-rapid antibiotic interventions. J Clin Microbiol. 2014:52(10):3805-7.

25. Peretz A, Isakovich N, Pastukh N, Koifman A, Glyatman T, Brodsky D. Performance of gram staining on blood cultures flagged negative by an automated blood culture system. Eur J Clin Microbiol Infect Dis. 2015;34(8): 1539-41.

\section{Publisher's Note}

Springer Nature remains neutral with regard to jurisdictional claims in published maps and institutional affiliations.

Ready to submit your research? Choose BMC and benefit from:

- fast, convenient online submission

- thorough peer review by experienced researchers in your field

- rapid publication on acceptance

- support for research data, including large and complex data types

- gold Open Access which fosters wider collaboration and increased citations

- maximum visibility for your research: over $100 \mathrm{M}$ website views per year

At BMC, research is always in progress.

Learn more biomedcentral.com/submissions 